\title{
Customized chemotherapy based on epidermal growth factor receptor mutation status for elderly patients with advanced non-small-cell lung cancer: a phase II trial
}

Shiro Fujita ${ }^{1 *}$, Nobuyuki Katakami ${ }^{1}$, Katsuhiro Masago ${ }^{2}$, Hiroshige Yoshioka ${ }^{3}$, Keisuke Tomii ${ }^{4}$, Toshihiko Kaneda ${ }^{5}$, Masataka Hirabayashi ${ }^{6}$, Kei Kunimasa ${ }^{3}$, Toshio Morizane ${ }^{7}$ and Tadashi Mio ${ }^{2}$

\begin{abstract}
Background: Elderly patients are more vulnerable to toxicity from chemotherapy. Activating epidermal growth factor receptor (EGFR) mutations in non-small-cell lung cancer (NSCLC) are associated with enhanced response to EGFR tyrosine-kinase inhibitors. We studied patients with advanced NSCLC for whom treatment was customized based on EGFR mutation status.

Methods: We screened 57 chemotherapy-naïve patients with histologically or cytologically confirmed NSCLC, stage IIIB or IV, aged 70 years or older, and with an Eastern Cooperative Oncology Group performance status 0 or 1, for EGFR exon 19 codon 746-750 deletion and exon 21 L858R mutation. Twenty-two patients with EGFR mutations received gefitinib; 32 patients without mutations received vinorelbine or gemcitabine. The primary endpoint was the response rate.

Results: The response rate was $45.5 \%$ (95\% confidence interval [Cl]: 24.4\%, 67.8\%) in patients with EGFR mutations and $18.8 \%$ (95\% Cl: $7.2 \%, 36.4 \%)$ in patients without EGFR mutations. The median overall survival was 27.9 months (95\% Cl: 24.4 months, undeterminable months) in patients with EGFR mutations and 14.9 months (95\% Cl: 11.0 months, 22.4 months) in patients without EGFR mutations. In the gefitinib group, grade 3/4 hepatic dysfunction and dermatitis occurred in $23 \%$ and $5 \%$ of patients, respectively. In patients treated with vinorelbine or gemcitabine, the most common grade 3 or 4 adverse events were neutropenia (47\%; four had febrile neutropenia), anemia (13\%), and anorexia (9\%). No treatment-related deaths occurred.

Conclusions: Treatment customization based on EGFR mutation status deserves consideration, particularly for elderly patients who often cannot receive second-line chemotherapy due to poor organ function or comorbidities.

Trial registration: This trial is registered at University hospital Medical Information Network-clinical trial registration (www.umin.ac.jp/ctr/index/htm) with the registration identification number C000000436.
\end{abstract}

\footnotetext{
* Correspondence: jp.shirofujita@gmail.com

${ }^{1}$ Institute of Biomedical Research and Innovation Hospital, 2-2 Minatojima

Minami-machi, Chuo-ku, Kobe 650-0047, Japan

Full list of author information is available at the end of the article
} 


\section{Background}

In many developed countries, lung cancer is the leading cause of cancer death for both men and women. Approximately $80 \%$ to $85 \%$ of lung cancer subtypes are nonsmall-cell lung cancer (NSCLC). Like other solid tumors, NSCLC primarily occurs in the elderly and most patients are diagnosed with advanced disease that is unsuitable for surgery [1,2]. As the geriatric population inexorably increases, more elderly patients with NSCLC will receive anticancer agents for palliative intent. Because definite potential exists for higher toxicity, attributed to progressive organ failure and comorbidities, single-agent chemotherapy (using a third-generation agent) along with platinum-based combinations is recommended for the treatment of this population [3,4].

Theoretically, drug-induced adverse events (AEs) are avoidable while maintaining anti-tumor effects by targeting the critical molecule that drives proliferation of and is solely present in cancer cells. After the success of imatinib for treatment of BCR-ABL-dependent chronic myelogenous leukemia $[5,6]$, understanding the molecular basis of human cancer has been exploited to provide targeted drugs. The epidermal growth factor receptor (EGFR) is a receptor tyrosine kinase (TK) of the ErbB family that has been implicated in cell proliferation and survival [7]. EGFR is a target of the tyrosine kinase inhibitor (TKI) gefitinib (Iressa; AstraZeneca, Macclesfield, United Kingdom), which has been approved for NSCLC treatment in many countries. The EGFR-TKIs achieved responses of approximately $10 \%$ in phase III clinical trials in patients with previously treated, unselected patients with advanced NSCLC $[8,9]$. Responses of EGFR-TKIs were more likely among certain patients: women, never- or light-smokers, patients with adenocarcinoma, and Asians [10-12]. In 2004, somatic mutations in the kinase domain of EGFR were predominantly found in these patients and were linked to EGFR-TKI sensitivity [13-15]. More than $90 \%$ of these mutations are observed in two hotspots: in-frame deletions including amino acids at codons 747 to 749 in exon 19, and an amino acid substitution at codon 858 (L858R) in exon 21 [16-18]. These mutations are postulated to mediate oncogenic effects by altering downstream signaling and anti-apoptotic mechanisms [13-15]. Prior trials confirmed that the response rate to EGFR-TKI in NSCLC patients with EGFR mutations is approximately 70$80 \%[12,19,20]$, and results of recent phase III trials showed that the oral EGFR-TKI gefitinib has a superior progression-free survival (PFS) to standard chemotherapy as the first-line therapy for NSCLC with mutated EGFR [21,22].

Mutations are found frequently in individuals of EastAsian ethnicity (30\%), and there are expected clinical benefits to gefitinib treatment in these patients [23]. For elderly patients with advanced NSCLC and wild-type $E G F R$, single-agent chemotherapy with a third-generation agent can be considered to be a recommended option. To maximize the effect of treatment, customized treatment based on EGFR mutation status would be mandatory. To evaluate the clinical feasibility and efficacy of such customized therapies, we conducted a prospective clinical trial that included patient assignment based on EGFR mutation status.

\section{Methods \\ Eligibility}

The study was a phase II, open-label, non-randomized, multicenter study (a phase II study of Iressa versus Vinorelbine or gemcitAbine in chemo-naïve elderly patients with advanced Non-small-cell lung cancer based on epidermal growth factor receptor mutation status: IVAN Trial; University hospital Medical Information Network-clinical trial registration number, C000000436). Patients enrolled in the present study had measurable, pathologically confirmed stage IIIB or IV NSCLC and were aged 70 years or older. Availability of archived tumor tissue or pleural/pericardial fluid for evaluation of EGFR mutation status was required for enrollment. Other eligibility criteria included written informed consent; no prior chemotherapy; Eastern Cooperative Oncology Group (ECOG) performance status (PS) 0 or 1; adequate bone marrow, renal, and hepatic function; and a life expectancy of at least 3 months. Exclusion criteria included symptomatic brain metastasis, any evidence of interstitial lung disease on chest computed tomography examination, other co-existing malignancies or malignancies diagnosed within the last 5 years other than carcinoma in situ, history of congestive heart failure, unstable angina pectoris or recent history (within 6 months) of acute myocardial infarction, uncontrolled cardiac arrhythmia, severe psychiatric illness, or concurrent disease or condition that would have made the patient inappropriate for study participation. The local ethical committees (the Research Ethics Committee of the Institute of Biomedical Research and Innovation, the Ethics Committee of Kyoto University Graduate School and Faculty of Medicine, the Clinical Research Approval Committee and the Medical Ethics Committee of Kurashiki Central Hospital, the Ethics Committee for Clinical Research of Kobe City Medical Center General Hospital, the Research Ethics Committee of Kobe City Medical Center West Hospital, and the institutional review board at the Hyogo Prefectural Amagasaki Hospital) approved the study, and written informed consent was obtained from each patient. The study was conducted in accordance with the Helsinki declaration. 


\section{EGFR mutation screening}

In the current open-label study, treatment was assigned based on EGFR mutation status. First, the EGFR gene mutational status of a biopsy specimen from each patient was evaluated. Initially, patients were screened for EGFR mutations in a commercial central laboratory at SRL in Tokyo. Both the exon 19 deletion mutation and the L858R point mutation were screened by direct sequencing, as described previously [13]. The T790M mutation, which was reported to be associated with resistance to EGFR-TKI therapy, was also checked, and the patient was excluded from the study if this mutation was detected [24]. From June 1, 2007, outsourcing of EGFR genetic testing was covered by government insurance in Japan, and the protocol was amended to allow the outsourcing from each institution to commercial clinical laboratories, either at Mitsubishi Chemical Medience in Tokyo (peptide nucleic acid-locked nucleic acid PCR clamp method) [25] or BML, Inc. in Tokyo (PCR-invader assay) [26]. During the study period, the methodology for EGFR mutation detection was substantially improved, and direct sequencing was substituted with highly-sensitive, advanced-generation detecting technology available in Japan.

\section{Treatment plan}

Patients whose tumor contained an EGFR activating mutation received gefitinib ( $250 \mathrm{mg}$ per day orally). In the present study, gefitinib was selected as the EGFR-TKI. Gefitinib was first approved in Japan for treatment of patients with advanced NSCLC on July 5th, 2002, and Japanese oncologists had already acquired considerable experience with this drug at the beginning of the present study. Patients with no EGFR activating mutation were treated with cytotoxic chemotherapy: either vinorelbine $25 \mathrm{mg} / \mathrm{m}^{2}$ on Day 1 and 8 (the recommended dose in Japan) or gemcitabine $1000 \mathrm{mg} / \mathrm{m}^{2}$ on Day 1 and 8 . The choice of the chemotherapy regimen was at the discretion of the attending physician, and a total of three to six cycles of treatment were required for either treatment regimen. Treatment was continued until progression of the disease (PD), development of unacceptable toxicity, patients' request, or completion of the treatment. Further therapy after PD was permitted in the protocol.

Dose modification of gefitinib, vinorelbine, or gemcitabine was allowed according to the protocol. The gefitinib dosing schedule could be modified to every second day for patients with severe toxicity: pulmonary toxicity (except for cough, forced expiratory volume in 1 second, hiccoughs, interstitial pneumonitis/pulmonary infiltrates) $\geq$ grade 2 , or grade $3 / 4$ diarrhea, mucositis, liver dysfunction, hematologic toxicity, skin toxicity, or ocular toxicity. For vinorelbine, a maximum of one dose reduction $\left(25\right.$ to $20 \mathrm{mg} / \mathrm{m}^{2}$ ) was allowed based on the following criteria: neutropenic fever, ileus $\geq$ grade 2 , nonhematological toxicity (except for nausea, hyponatremia, body weight loss, anorexia, and alopecia) $\geq$ grade 3 , grade 4 neutropenia and/or thrombocytopenia. Regarding gemcitabine, a maximum of one dose reduction (1000 to $800 \mathrm{mg} / \mathrm{m}^{2}$ ) was allowed based on the following criteria: neutropenic fever, interstitial pneumonitis or pulmonary fibrosis $\geq$ grade 1 , non-hematological toxicity (except for nausea, hyponatremia, body weight loss, anorexia, and alopecia) $\geq$ grade 3 , grade 4 neutropenia and/or thrombocytopenia.

The primary outcome of the study was the objective response rate (ORR): partial plus complete response based on the Response Evaluation Criteria in Solid Tumors [27]. The present study was designed to detect a response rate of $65 \%$ in the gefitinib group compared with a minimal, clinically meaningful response rate of $30 \%$. It was estimated before starting the present study that 20 eligible patients would allow the study to have $80 \%$ power to detect this difference, with an alpha level of 0.05 . The drop rate was considered to be $10 \%$, and a total of 22 patients would need to be enrolled in the gefitinib group. We estimated that the frequency of NSCLC with EGFR mutations accounted for $25 \%$ of the total NSCLC population, based on the results of the preprotocol survey regarding the number of newly referred advanced NSCLC patients to two hospitals (Kyoto University Hospital and the Institute of Biomedical Research and Innovation Hospital; data not shown). To enroll 22 patients in the gefitinib group, 88 patients would need to be screened, and 66 of the 88 patients were expected to have tumors with wild-type EGFR. To detect a response rate of $20 \%$ compared with a minimal, clinically meaningful response rate of $5 \%$ in the single-agent chemotherapy group, 27 patients were required to maintain more than $80 \%$ power to detect this difference, with an alpha level of 0.05 . Sixty-six patients, estimated in the pre-protocol period, were sufficient. We expected that patients who had predictive factors for containing an EGFR mutation (i.e. female, East-Asian origin, adenocarcinoma, and no history of smoking) were more likely to be recruited in our trial. In other words, patients with wild-type EGFR would be less likely to be enrolled. In this case, 27 patients were minimally required to detect the difference in the single-agent chemotherapy group. The drop rate was considered to be $10 \%$, and a total of 30 patients would need to be enrolled in the single-agent chemotherapy group.

Planned secondary outcomes included the disease control rate, 1-year survival rate, overall survival (OS), time to treatment failure (TTF), and toxicity. Disease control was defined as the best response out of complete response, partial response, or stable disease, which was 
confirmed and sustained for 4 weeks or longer. TTF was measured from the start date of treatment to the date of discontinuation of the study treatment, occurrence of $\mathrm{PD}$, or death by any cause (whichever occurred earlier). If intolerable toxicity or discontinuation of treatment secondary to toxicity occurred, the patient was considered assessable but was classified as a treatment failure.

During treatment, assessments were performed every 6 weeks until disease progression. Events were confirmed twice via source-document verification at site visits or delivered radiographic data by members of the data center and the investigators.

Safety was monitored by clinical AEs, laboratory (hematology and clinical chemistry) testing, radiographic information, and collection of vital signs, weight, and ECOG PS status. AE severity was graded based on the National Cancer Institute Common Toxicity Criteria (version 3.0). Interstitial pneumonitis was the most serious concern related to gefitinib treatment because this event has been noted in patients taking gefitinib.

OS and TTF were estimated by the Kaplan-Meier method, and the therapy arms were compared by logrank test. Categorical variables were analyzed by Fisher's exact test or $X^{2}$ test. Continuous variables were assessed with unpaired $t$-tests or the Mann-Whitney $U$-test. Statistical analyses were performed using JMP version 6 (SAS Institute, Cary, NC, USA) and R software version 2.8.1 (R Foundation for Statistical Computing, Vienna, Austria).

\section{Results}

Accrual

Enrollment started in July 2006. From June 2007, the rate of enrollment slowed, mainly because government insurance coverage of EGFR genetic analysis had begun in Japan, and the benefit of enrollment in the present study was substantially lessened. The last patient was enrolled in April 2009. The data cut-off date used for the present report was February 10, 2011.

\section{Patients}

Fifty-seven patients were screened from July 2006 to April 2009 (Figure 1). Mutational analysis for one patient was unsuccessful because the amount of the specimen was insufficient. During analyses, two patients suffered serious illnesses not related to their cancer (acute atherothrombotic ischemic stroke of the right cerebrum in one patient and congestive heart failure due to constrictive pericarditis in another patient). These patients were deemed ineligible. Of the remaining 54 patients, 22 patients (41\%) harbored mutations and 32 patients' tumors were wild type. There was no patient whose tumor revealed the T790M mutation in exon 20, and all 54 patients were enrolled in the study. The

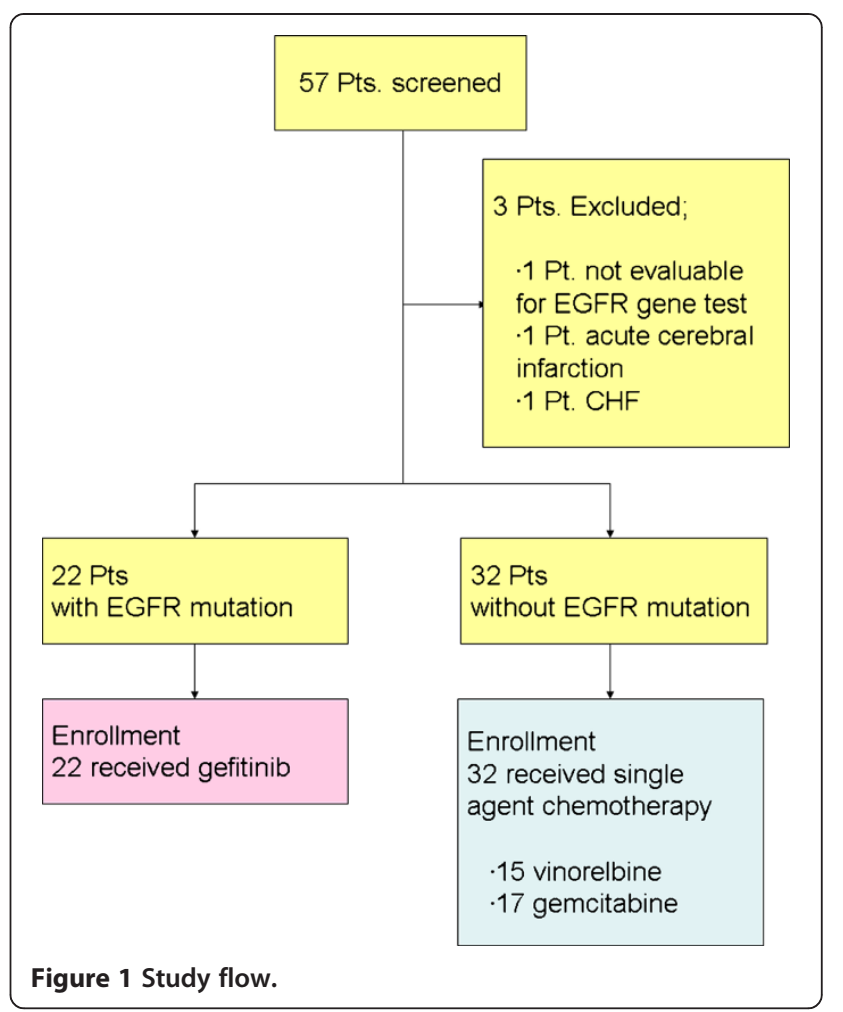

demographic information of the patients is summarized in Table 1. As expected, some imbalances were observed between the treatment groups; more never-smokers (73\% vs. $41 \%)$ and patients with adenocarcinoma histology (95\% vs. $50 \%)$ were in the gefitinib group. These imbalances seemed to reflect the epidemiologic features of NSCLC harboring activating EGFR mutations. At the data cut-off, the median follow up was 15.8 months.

\section{Response and survival of the gefitinib group}

A summary of the tumor response is shown in Table 2 . The ORR was $45.5 \%$ (95\% confidence interval [CI], 24.4-67.8\%) and disease control rate was $86.4 \%$ (95\% CI, 65.1-97.1\%) for the gefitinib group. Eleven patients had died among the gefitinib group. The Kaplan-Meier estimate of the median TTF was 9.7 months (Figure 2) and the median survival time (MST) was 27.9 months (Figure 3). The 1-year OS rate was $90.0 \%$.

\section{Response and survival of the vinorelbine/gemcitabine group}

As shown in Table 2, the ORR was $18.8 \%$ (95\% CI, 7.2$36.4 \%$ ) and the disease control rate was $56.3 \%$ (95\% CI, $37.7-73.6 \%$ ) for the vinorelbine (VNR)/gemcitabine (GEM) group. Twenty-two patients had died among the VNR/GEM group. The Kaplan-Meier estimate of the median TTF was 2.9 months (Figure 4) and MST was 14.9 months (Figure 5). The 1-year OS rate was $60.1 \%$. 
Table 1 Patient characteristics

\begin{tabular}{|c|c|c|c|c|}
\hline & All & Gefitinib & VNR or GEM & $P$ \\
\hline \multicolumn{5}{|l|}{ Age (years) } \\
\hline Median (range) & 80 (71-89) & $81(71-85)$ & 79 (72-89) & 0.11 \\
\hline Male:Female & $20: 34$ & $5: 17$ & $15: 17$ & 0.09 \\
\hline \multicolumn{5}{|l|}{ ECOG PS } \\
\hline $0: 1$ & $12: 42$ & $8: 14$ & $4: 28$ & 0.05 \\
\hline \multicolumn{5}{|l|}{ Histology } \\
\hline Adeno & 37 & 21 & 16 & $<0.001$ \\
\hline Squamous & 8 & 1 & 7 & \\
\hline Other & 9 & 0 & 9 & \\
\hline \multicolumn{5}{|l|}{ Smoking history } \\
\hline Never & 29 & 16 & 13 & 0.03 \\
\hline Former / current & 25 & 6 & 19 & \\
\hline \multicolumn{5}{|l|}{ Mutation type } \\
\hline Exon 19 del & 6 & 6 & - & - \\
\hline Exon 21 L858R & 14 & 14 & - & \\
\hline Ex19del + L858R & 2 & 2 & - & \\
\hline \multicolumn{5}{|l|}{ Clinical stage } \\
\hline IIIB & 20 & 9 & 11 & 0.78 \\
\hline IV & 34 & 13 & 21 & \\
\hline
\end{tabular}

ECOG PS: Eastern Cooperative Oncology Group Performance Status; GEM: gemcitabine; VNR: vinorelbine.

\section{Comparison with regard to efficacy between the two groups}

The baseline characteristics were different between the gefitinib and VNR/GEM groups because of the nonrandomized nature of the study; however, a statistically significant difference was found regarding the disease control rate $(86.4 \%$ vs. $56.3 \% ; P=0.035)$, but not regarding the ORR $(45.5 \%$ vs. $18.8 \% ; P=0.067)$. Concerning the TTF, a statistically significant difference was observed between the groups (log-rank test; $P=0.0008$; Figure 6). Additionally, a significantly longer OS was noted in the gefitinib group compared with the VNR/ GEM group (log-rank test; $P=0.016$; Figure 7 ), although there was a distinct difference between tumors in each

Table 2 Best response according to RECIST in both groups

\begin{tabular}{lll}
\hline & Gefitinib $(\mathbf{N}=\mathbf{2 2})$ & VNR or GEM $(\mathbf{N}=\mathbf{3 2})$ \\
\hline Complete response & 0 & 0 \\
Partial response & $10(45.5 \%)$ & $6(18.8 \%)$ \\
Stable disease & $9(40.9 \%)$ & $12(37.5 \%)$ \\
Progressive disease & $1(4.5 \%)$ & $6(18.8 \%)$ \\
Not evaluable & $2(9.1 \%)$ & $8(25.0 \%)$ \\
\hline
\end{tabular}

GEM: gemcitabine; RECIST: Response Evaluation Criteria in Solid Tumors; VNR: vinorelbine.

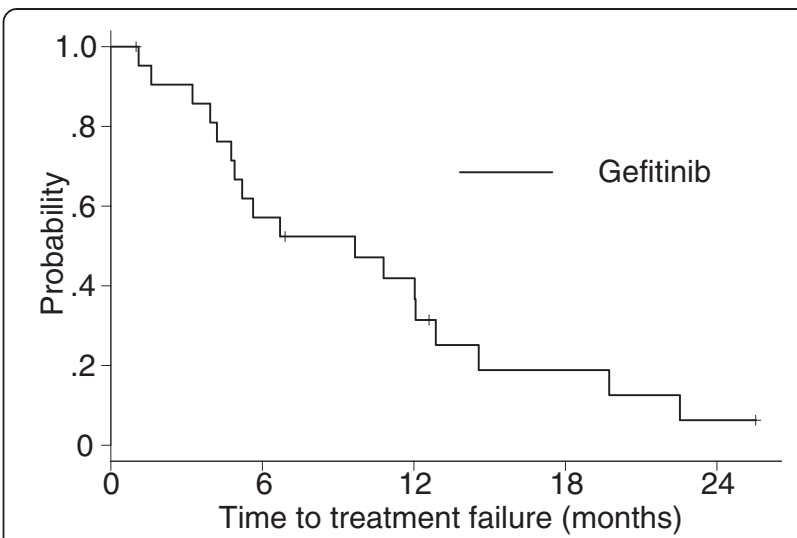

Figure $\mathbf{2}$ Time to treatment failure of the gefitinib group.

group with regard to the EGFR mutation status. For all patients, the MST was 19.7 months (Figure 8).

\section{Second-line therapy}

Of the 22 patients assigned to gefitinib, 14 received chemotherapy, 5 received radiotherapy, and 8 received EGFR-TKI (six received erlotinib, two underwent rechallenge with gefitinib); 2 received no therapy poststudy. Of the 32 patients assigned to single-agent chemotherapy, 14 received second-line chemotherapy, 8 received radiotherapy, and 15 patients received EGFRTKI (nine received erlotinib and six received gefitinib); five patients received no therapy post-study. No patient in the single-agent chemotherapy group was in the protocol-treatment phase.

\section{Toxicity}

Toxicity information was available for all 54 treated patients. Treatment-related AEs were observed in 52 of the 54 treated patients. No treatment-related deaths occured in the gefitinib or VNR/GEM groups. AEs occurring in more than $10 \%$ of either treatment group are listed in Table 3. In the gefitinib group, skin-related AEs

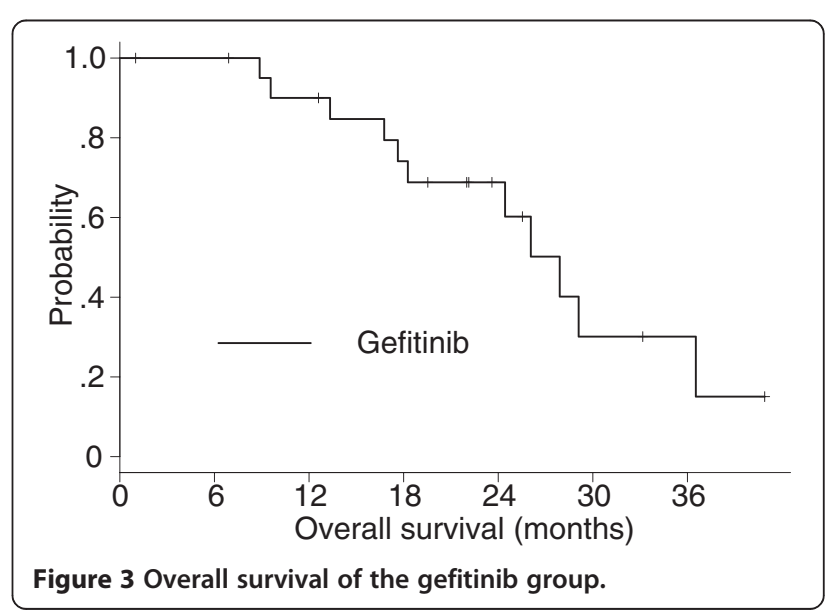




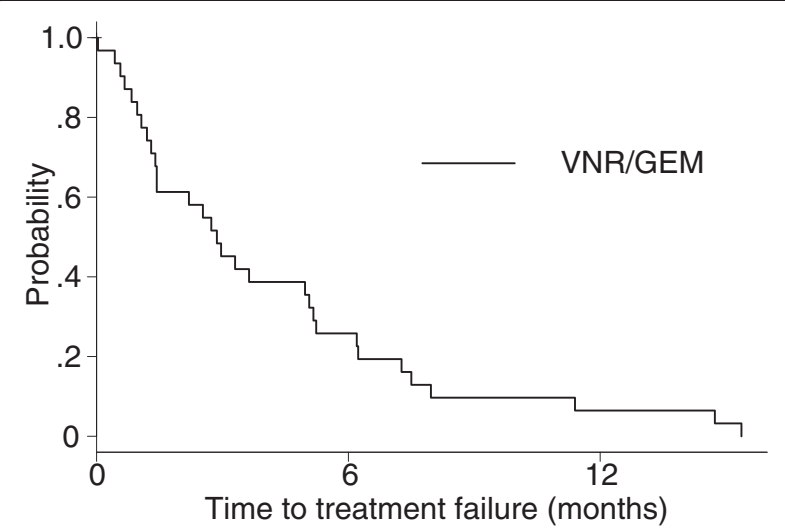

Figure 4 Time to treatment failure of the chemotherapy group.

were observed in 14 of 22 patients (64\%), and the most frequently occurring grade 3 or worse $\mathrm{AE}$ was elevation of aminotransferase. No interstitial lung disease was observed. Eleven patients (52\%) in the gefitinib group had at least one drug discontinuation due to AEs; elevation of aminotransferases in six patients and skin toxicity in five patients.

As expected, hematologic AEs were common in the VNR/GEM group (Table 3). Grade 3 or 4 neutropenia, anemia, and thrombocytopenia occurred in 15 patients (47\%), four patients (13\%), and one patient (3\%), respectively. Febrile neutropenia was observed in four patients (13\%). The most common grade 3 or 4 non-hematologic $\mathrm{AE}$ in the VNR/GEM group was anorexia followed by fatigue, nausea, and hyponatremia. Dose reduction or treatment delay was documented in 14 patients $(44 \%)$ due to neutropenia or neutropenic fever (eight patients), fever (three patients), patients' request (two patients), or grade 3 constipation (one patient).

\section{Discussion}

In the present study, the median TTF and MST of patients with mutated EGFR (treated with gefitinib) were

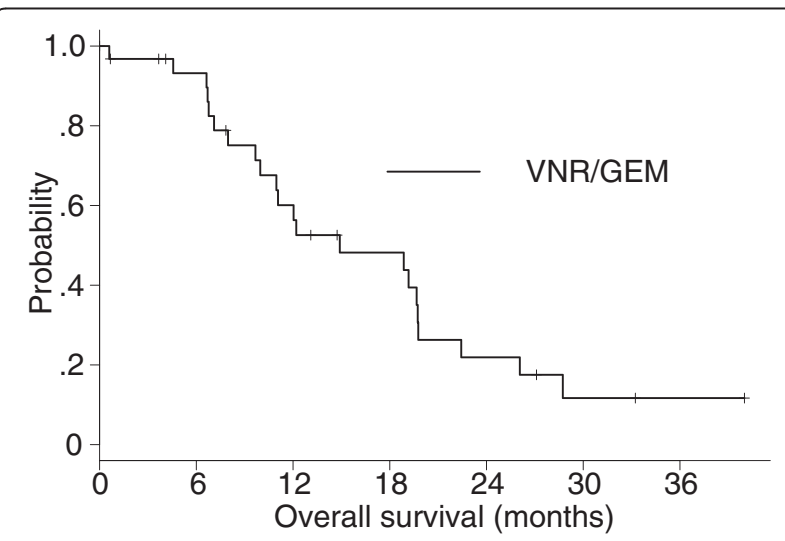

Figure 5 Overall survival of the chemotherapy group.

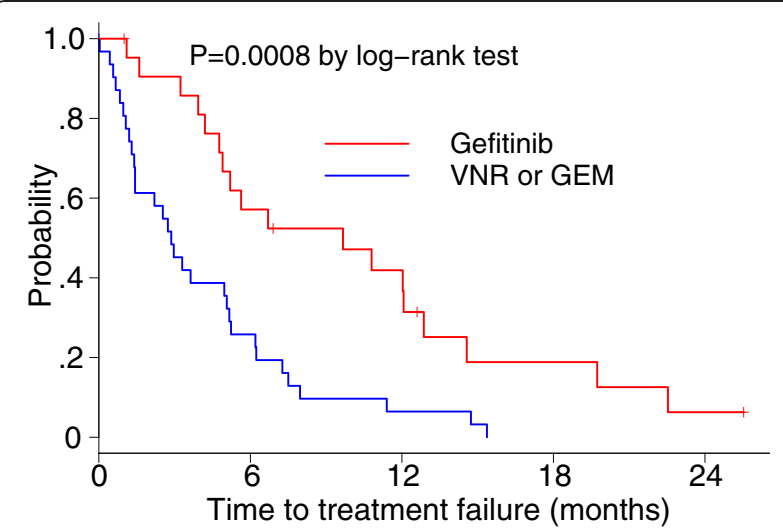

Figure 6 Kaplan-Meier curve of TTF according to the treatment group.

9.7 months and 27.9 months, respectively, which were comparable to the results of two recent trials. The North East Japan 002 Gefitinib Study Group conducted a phase III trial comparing an EGFR mutation-positive group of patients receiving gefitinib with carboplatin plus paclitaxel [21]. In the planned interim analysis, the PFS was significantly longer in the gefitinib group and the study was terminated. The gefitinib group had a significantly longer PFS compared with the chemotherapy group (10.8 months vs. 5.4 months, respectively; hazard ratio, $0.30 ; P<0.001)$. The MST for the gefitinib group was 30.5 months. Mitsudomi et al., from the West Japan Thoracic Oncology Group, compared gefitinib with cisplatin plus docetaxel as the first-line treatment for advanced NSCLC with EGFR mutations [22]. The gefitinib group had a significantly longer PFS compared with the cisplatin plus docetaxel group, with a median PFS of 9.2 months vs. 6.3 months, respectively (hazard ratio, 0.489; $P<0.0001)$. These results represent a milestone toward "tailor-made" therapy for NSCLC and gefitinib

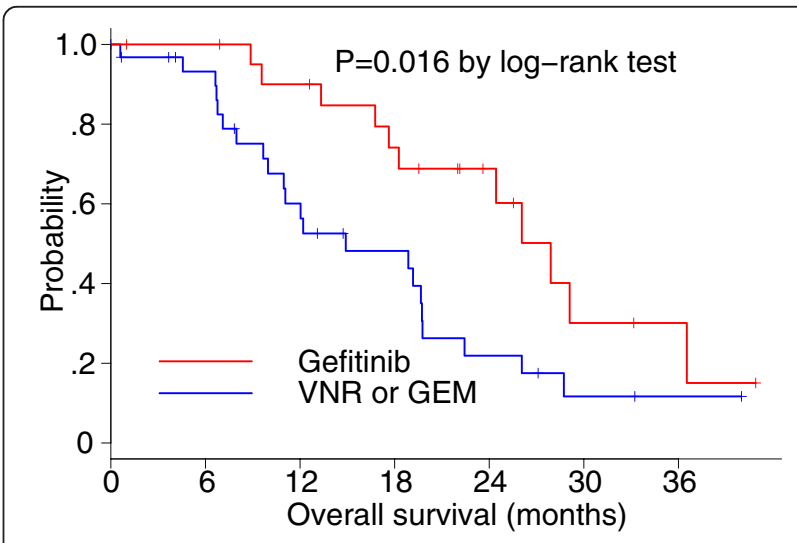

Figure 7 Kaplan-Meier curve of OS according to the treatment group. 


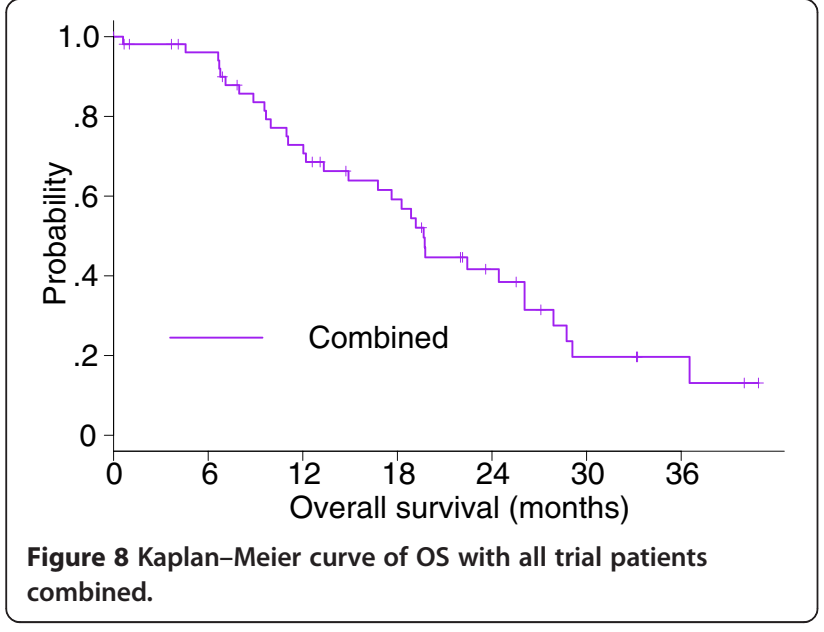

has been recently registered as the first-line treatment for NSCLC patients with EGFR activating mutations in Europe. Elderly patients have less of a chance of receiving second-line chemotherapy compared with their younger counterparts, due to poor organ function and/or comorbidities [28], and many elderly patients with relapsed or refractory NSCLC after first-line chemotherapy are deemed unfit for second-line chemotherapy [29]. Upfront administration of the most effective regimen (i.e., a regimen that is expected to have the longest PFS) is needed for these patients. The use of first-line gefitinib is valuable for elderly patients with EGFR mutant NSCLC.

No significant unexpected toxicity was found in the gefitinib group. Similar to other EGFR-targeted agents, skin-related AEs were the most common toxicity observed. No interstitial lung disease was reported. The Grade 3 to 4 elevation of alanine aminotransferase (ALT) and aspartate aminotransferase (AST) was observed in $22.7 \%$ and $13.6 \%$ of patients who received gefitinib, respectively. This result was comparable to previous studies conducted in Japan. The most common toxicity criteria (CTC)-AE grade 3 or 4 toxicity reported in the gefitinib group of the North East Japan 002 trial was aminotransferase elevation (26.3\%) [21]. In the West Japan Thoracic Oncology Group 3405 trial, 14 of 87 patients (16.1\%) experienced CTC-AE grade 3 or 4 AST elevation, and grade 3 or 4 ALT elevation was observed in 24 of 87 patients (27.6\%) [22]. This liver enzyme elevation is almost always reversible; however, it may cause a decrease in dose intensity and may lessen efficacy.

The ORR in the gefitinib group was $45 \%$, which seemed to be low compared with that reported in the literature $[12,19,20]$. One cause may be the high drugdiscontinuation rate due to AEs. In the gefitinib group, 11 patients (50\%) had drug discontinuation and dose intensity was lessened, which explains the low ORR in the gefitinib group. In the present study, all participants
Table 3 Adverse events (AEs) occurring in more than $10 \%$ of either treatment group

\begin{tabular}{lll}
\hline Gefitinib $(\mathbf{N}=\mathbf{2 2})$ & All Grades & CTC Grade $\mathbf{3}$ or $\mathbf{4}$ \\
\hline Skin-related AEs & $14(63.6 \%)$ & $1(4.5 \%)$ \\
ALT & $14(63.6 \%)$ & $5(22.7 \%)$ \\
AST & $12(54.5 \%)$ & $3(13.6 \%)$ \\
Anemia & $11(50.0 \%)$ & 0 \\
Hypoalbminemia & $9(40.9 \%)$ & 0 \\
Anorexia & $6(27.3 \%)$ & 0 \\
Fatigue & $6(27.3 \%)$ & 0 \\
Diarrhea & $6(27.3 \%)$ & 0 \\
ALP & $6(27.3 \%)$ & 0
\end{tabular}

VNR or GEM $(\mathbf{N}=\mathbf{3 2})$

All Grades

CTC Grade 3 or 4

Anemia

Leukocytopenia

$23(71.9 \%) \quad 4(12.5 \%)$

Neutropenia

$21(65.6 \%)$

$15(46.9 \%)$

Fatigue

$20(62.5 \%)$

$15(46.9 \%)$

$18(56.3 \%)$

$2(6.3 \%)$

Anorexia

$12(37.5 \%)$

$3(9.4 \%)$

Thrombocytopenia

ALT

Nausea

$12(37.5 \%)$

$1(3.1 \%)$

$12(37.5 \%)$

0

$11(34.4 \%)$

$2(6.3 \%)$

Constipation

AST

Hyponatremia

$9(28.1 \%)$

$1(3.1 \%)$

$9(28.1 \%)$

0

Hyperkalemia

Hypocalcemia

$5(15.6 \%)$

$2(6.3 \%)$

Febrile neutropenia

$5(15.6 \%)$

0

$5(15.6 \%)$

0

AE: adverse event; ALT: alanine aminotransferase; ALP: alkaline phosphatase; AST: aspartate aminotransferase; CTC: common toxicity criteria; GEM: gemcitabine; VNR: vinorelbine.

were aged 70 years or older, and the median age of the enrolled patients was 80 in the entire treatment population. Age-related decreases in organ function have the potential to increase drug-related toxicity in the elderly, although chronological age itself should not preclude appropriate treatment. Given the favorable TTF and OS in the gefitinib group, gefitinib was just as effective in the elderly with mutated EGFR compared with their younger counterparts. A comprehensive guide on dose adjustments in this population is mandatory, even for this recently developed, molecular-targeted agent.

Monotherapy with vinorelbine, gemcitabine, or docetaxel is still reasonable for patients with NSCLC of unknown EGFR mutation status (i.e., both EGFR mutation-positive and -negative groups combined). In the key phase III study ELVIS (the Elderly Lung Cancer Vinorelbine Italian Study) [30,31], 161 chemotherapynaïve patients ( $\geq 70$ years old) were randomized to receive vinorelbine or best supportive care. The ORR was $20 \%$ and 1-year survival rate was $32 \%$ for patients 
receiving vinorelbine, and a significant survival benefit was recorded compared with the control group. In another key phase III study, the Multicenter Italian Lung Cancer in the Elderly Study, 700 patients were randomized to receive single-agent chemotherapy with vinorelbine or gemcitabine, or combination therapy with vinorelbine plus gemcitabine [32]. Combination treatment had no advantage regarding ORR, time to progression (TTP), or survival over single-agent therapy. The ORR was $18 \%, 16 \%$, and $21 \%$ for vinorelbine, gemcitabine, and vinorelbine plus gemcitabine, respectively; the median TTP was $4.5,4.3$, and 4.8 months and MST was 9.0, 7.0, and 7.5 months, respectively. A phase III trial (West Japan Thoracic Oncology Group 9904), which compared docetaxel with vinorelbine for the treatment of elderly patients with advanced NSCLC, demonstrated a statistically significant improvement in median PFS (5.5 months vs. 3.1 months, respectively) and ORR ( $22.7 \%$ vs. $9.9 \%$, respectively; $P=0.019$ ), but no statistical difference was found regarding the MST (14.3 months vs. 9.9 months, respectively; $P=0.138$ ) [29]. Recently, the IFCT-0501 phase III study demonstrated an improved OS of a platinum-based combination regimen (carboplatin plus paclitaxel) compared with single-agent chemotherapy; however, toxicity was an issue, as 9 of 143 (6.3\%) patients died due to treatment-related AEs [33]. While the survival benefit with doublet chemotherapy is impressive, accompanying toxicity is a concern with this regimen.

In our study, single-agent chemotherapy was effective and feasible for the elderly patients with NSCLC who were wild type for EGFR. The ORR of $18.8 \%$ and MST of 14.9 months observed in the single agent chemotherapy group were compatible to the results of the previous clinical trials mentioned above. The major AEs were related to myelosuppression, were clinically manageable, and included no treatment-related deaths. Given the favorable results, we suggest that cytotoxic agent monotherapy should be considered to be the treatment for elderly patients with NSCLC who are wild type for EGFR.

At first, we planned a subsequent phase III study to evaluate the customized treatment based on EGFR mutation status, with patients randomized to either a control (conventional cytotoxic chemotherapy) arm or experimental (customized treatment) arm. In June 2007, however, outsourcing of EGFR genetic testing was fully covered by the health-insurance system in Japan, and customized treatment based on EGFR mutation status is a part of the practice for the treatment of NSCLC. Advanced-generation analytical methods, including highly-specific, rapid PCR techniques, allow us to detect EGFR mutations from fluid specimens, such as malignant effusion or aspiration-needle fluid, with satisfactory sensitivity and specificity. Most of patients with NSCLC have their tumor analyzed when the pathological diagnosis is confirmed, and the results of EGFR mutational analysis are usually available at the beginning of the first-line chemotherapy. Results of the present study represent outcomes of the current standard therapy for elderly patients with NSCLC in Japan, although the number of enrolled patients was small.

\section{Conclusions}

This is the first prospective multi-center study in the elderly population with advanced NSCLC that included customized treatment based on EGFR mutation status. The MST of all participants (both groups combined) was 19.7 months, which was favorable in elderly patients (aged 70 years or older) with advanced NSCLC.

\section{Competing interests}

The authors declare that they have no competing or financial interests.

\section{Acknowledgements}

This work is supported by Health Promoting Association for Respiratory Medicine of Nishi-Nippon. This manuscript was written on behalf of the IVAN Trial investigators. The authors acknowledge Dr. Morgan Tucker for his assistance in writing the manuscript. The authors would like to thank all the patients who participated in this study and their family.

\section{Author details}

${ }^{1}$ Institute of Biomedical Research and Innovation Hospital, 2-2 Minatojima Minami-machi, Chuo-ku, Kobe 650-0047, Japan. ${ }^{2}$ Kyoto University Hospital, 54 Shogoin Kawaracho, Sakyo-ku, Kyoto 606-8507, Japan. ${ }^{3}$ Kurashiki Central Hospital, 1-1-1 Miwa, Kurashiki-shi, Okayama 710-8602, Japan. ${ }^{4}$ Kobe City Medical Center General Hospital, 2-1-1 Minatojima Minami-machi, Chuo-ku, Kobe 650-0047, Japan. ${ }^{5}$ Kobe City Medical Center West Hospital, 2-4 Ichibancho, Nagata-ku, Kobe 653-0013, Japan. ${ }^{6}$ Hyogo Prefectural Amagasaki Hospital, Higashidaimotsu-cho, Amagasaki, Hyogo 660-0828, Japan. ${ }^{7}$ Kanagawa Dental College, 82 Inaokacho, Yokosuka-ku, Kanagawa 238-8580, Japan.

\section{Authors' contributions}

SF and T. Mio designed the study. SF, NK, KM, HY, KT, TK, and MH contributed with provision of study materials or patients. SF, NK, KM, HY, KK, $\mathrm{KT}, \mathrm{TK}, \mathrm{MH}$, and T. Mio contributed with collection and assembly of data. SF, NK, and T. Morizane performed statistical data analysis and helped with data interpretation. SF, NK, KM, HY, KK, KT, TK, MH, and T. Mio drafted the

manuscript. All authors read and approved the final manuscript.

Received: 13 October 2011 Accepted: 21 May 2012

Published: 21 May 2012

\section{References}

1. Edwards BK, Howe HL, Ries LA, Thun MJ, Rosenberg HM, Yancik R, Wingo PA, Jemal A, Feigal EG: Annual report to the nation on the status of cancer, 1973-1999, featuring implications of age and aging on U.S. cancer burden. Cancer 2002, 94(10):2766-2792.

2. Owonikoko TK, Ragin CC, Belani CP, Oton AB, Gooding WE, Taioli E, Ramalingam SS: Lung cancer in elderly patients: an analysis of the surveillance, epidemiology, and end results database. J Clin Oncol 2007, 25(35):5570-5577.

3. NCCN Clinical Practice Guidelines in Oncology (NCCN Guidelines) Version 1.2011.: ; 2010.

4. D'Addario G, Fruh M, Reck M, Baumann P, Klepetko W, Felip E: Metastatic non-small-cell lung cancer: ESMO Clinical Practice Guidelines for diagnosis, treatment and follow-up. Ann Oncol 2010, 21(Suppl 5):v116-v119.

5. O'Brien SG, Guilhot F, Larson RA, Gathmann I, Baccarani M, Cervantes F, Cornelissen JJ, Fischer T, Hochhaus A, Hughes T, et al: Imatinib compared 
with interferon and low-dose cytarabine for newly diagnosed chronicphase chronic myeloid leukemia. N Engl J Med 2003, 348(11):994-1004.

6. Druker BJ, Guilhot F, O'Brien SG, Gathmann I, Kantarjian H, Gattermann N, Deininger MW, Silver RT, Goldman JM, Stone RM, et al: Five-year follow-up of patients receiving imatinib for chronic myeloid leukemia. $N$ Engl J Med 2006, 355(23):2408-2417.

7. Mendelsohn J: Blockade of receptors for growth factors: an anticancer therapy-the fourth annual Joseph $\mathrm{H}$ Burchenal American Association of Cancer Research Clinical Research Award Lecture. Clin Cancer Res 2000 6(3):747-753.

8. Shepherd FA, Rodrigues Pereira J, Ciuleanu T, Tan EH, Hirsh V, Thongprasert S, Campos D, Maoleekoonpiroj S, Smylie M, Martins R, et al: Erlotinib in previously treated non-small-cell lung cancer. N Engl J Med 2005, 353(2):123-132.

9. Thatcher N, Chang A, Parikh P, Rodrigues Pereira J, Ciuleanu T, von Pawel J, Thongprasert S, Tan EH, Pemberton K, Archer V, et al: Gefitinib plus best supportive care in previously treated patients with refractory advanced non-small-cell lung cancer: results from a randomised, placebocontrolled, multicentre study (Iressa Survival Evaluation in Lung Cancer). Lancet 2005, 366(9496):1527-1537.

10. Fukuoka M, Yano S, Giaccone G, Tamura T, Nakagawa K, Douillard JY, Nishiwaki Y, Vansteenkiste J, Kudoh S, Rischin D, et al: Multi-institutional randomized phase II trial of gefitinib for previously treated patients with advanced non-small-cell lung cancer (The IDEAL 1 Trial) [corrected]. J Clin Oncol 2003, 21(12):2237-2246.

11. Kris MG, Natale RB, Herbst RS, Lynch TJ Jr, Prager D, Belani CP, Schiller JH, Kelly K, Spiridonidis H, Sandler A, et al: Efficacy of gefitinib, an inhibitor of the epidermal growth factor receptor tyrosine kinase, in symptomatic patients with non-small cell lung cancer: a randomized trial. JAMA 2003, 290(16):2149-2158

12. Mok TS, Wu YL, Thongprasert $S$, Yang CH, Chu DT, Saijo N, Sunpaweravong $P$, Han B, Margono B, Ichinose $Y$, et al: Gefitinib or carboplatin-paclitaxel in pulmonary adenocarcinoma. N Engl J Med 2009, 361(10):947-957.

13. Lynch TJ, Bell DW, Sordella R, Gurubhagavatula S, Okimoto RA, Brannigan BW, Harris PL, Haserlat SM, Supko JG, Haluska FG, et al: Activating mutations in the epidermal growth factor receptor underlying responsiveness of non-small-cell lung cancer to gefitinib. N Engl J Med 2004, 350(21):2129-2139.

14. Paez JG, Janne PA, Lee JC, Tracy S, Greulich H, Gabriel S, Herman P, Kaye FJ, Lindeman N, Boggon TJ, et al: EGFR mutations in lung cancer: correlation with clinical response to gefitinib therapy. Science 2004, 304(5676):1497-1500.

15. Pao W, Miller V, Zakowski M, Doherty J, Politi K, Sarkaria I, Singh B, Heelan R, Rusch $V$, Fulton $L$, et al: EGF receptor gene mutations are common in lung cancers from "never smokers" and are associated with sensitivity of tumors to gefitinib and erlotinib. Proc Natl Acad Sci U S A 2004, 101 (36):13306-13311.

16. Kosaka T, Yatabe $Y$, Endoh H, Kuwano H, Takahashi T, Mitsudomi T: Mutations of the epidermal growth factor receptor gene in lung cancer: biological and clinical implications. Cancer Res 2004, 64(24):8919-8923.

17. Takano T, Ohe Y, Sakamoto H, Tsuta K, Matsuno Y, Tateishi U, Yamamoto S, Nokihara H, Yamamoto N, Sekine I, et al: Epidermal growth factor receptor gene mutations and increased copy numbers predict gefitinib sensitivity in patients with recurrent non-small-cell lung cancer. J Clin Oncol 2005, 23(28):6829-6837.

18. Rosell R, Moran T, Queralt C, Porta R, Cardenal F, Camps C, Majem M, LopezVivanco G, Isla D, Provencio $M$, et al: Screening for epidermal growth factor receptor mutations in lung cancer. N Engl J Med 2009, 361(10):958967.

19. Morita S, Okamoto I, Kobayashi K, Yamazaki K, Asahina H, Inoue A, Hagiwara K, Sunaga N, Yanagitani N, Hida T, et al: Combined survival analysis of prospective clinical trials of gefitinib for non-small cell lung cancer with EGFR mutations. Clin Cancer Res 2009, 15(13):4493-4498.

20. Sequist LV, Martins RG, Spigel D, Grunberg SM, Spira A, Janne PA, Joshi VA, McCollum D, Evans TL, Muzikansky A, et al: First-line gefitinib in patients with advanced non-small-cell lung cancer harboring somatic EGFR mutations. J Clin Oncol 2008, 26(15):2442-2449.

21. Maemondo M, Inoue A, Kobayashi K, Sugawara S, Oizumi S, Isobe H, Gemma A, Harada M, Yoshizawa H, Kinoshita I, et al: Gefitinib or chemotherapy for non-small-cell lung cancer with mutated EGFR. N Engl J Med 2010, 362(25):2380-2388.
22. Mitsudomi T, Morita S, Yatabe Y, Negoro S, Okamoto I, Tsurutani J, Seto T, Satouchi M, Tada H, Hirashima T, et al: Gefitinib versus cisplatin plus docetaxel in patients with non-small-cell lung cancer harbouring mutations of the epidermal growth factor receptor (WJTOG3405): an open label, randomised phase 3 trial. Lancet Oncol 2010, 11(2):121-128.

23. Mitsudomi T, Yatabe $Y$ : Mutations of the epidermal growth factor receptor gene and related genes as determinants of epidermal growth factor receptor tyrosine kinase inhibitors sensitivity in lung cancer. Cancer Sci 2007, 98(12):1817-1824.

24. Kobayashi S, Boggon TJ, Dayaram T, Janne PA, Kocher O, Meyerson M, Johnson BE, Eck MJ, Tenen DG, Halmos B: EGFR mutation and resistance of non-small-cell lung cancer to gefitinib. N Engl J Med 2005, 352(8):786-792.

25. Nagai Y, Miyazawa H, Huqun ZZ, Tanaka T, Udagawa K, Kato M, Fukuyama S, Yokote A, Kobayashi K, Kanazawa M, et al: Genetic heterogeneity of the epidermal growth factor receptor in non-small cell lung cancer cell lines revealed by a rapid and sensitive detection system, the peptide nucleic acid-locked nucleic acid PCR clamp. Cancer Res 2005, 65(16):7276-7282

26. Lyamichev V, Mast AL, Hall JG, Prudent JR, Kaiser MW, Takova T, Kwiatkowski RW, Sander TJ, de Arruda M, Arco DA, et al: Polymorphism identification and quantitative detection of genomic DNA by invasive cleavage of oligonucleotide probes. Nat Biotechnol 1999, 17(3):292-296.

27. Therasse P, Arbuck SG, Eisenhauer EA, Wanders J, Kaplan RS, Rubinstein L, Verweij J, Van Glabbeke M, van Oosterom AT, Christian MC, et al: New guidelines to evaluate the response to treatment in solid tumors. European Organization for Research and Treatment of Cancer, National Cancer Institute of the United States, National Cancer Institute of Canada. J Natl Cancer Inst 2000, 92(3):205-216.

28. Davidoff AJ, Tang M, Seal B, Edelman MJ: Chemotherapy and survival benefit in elderly patients with advanced non-small-cell lung cancer. $J$ Clin Oncol 2010, 28(13):2191-2197.

29. Kudoh S, Takeda K, Nakagawa K, Takada M, Katakami N, Matsui K, Shinkai T, Sawa T, Goto I, Semba H, et al: Phase III study of docetaxel compared with vinorelbine in elderly patients with advanced non-small-cell lung cancer: results of the West Japan Thoracic Oncology Group Trial (WJTOG 9904). J Clin Oncol 2006, 24(22):3657-3663.

30. The Elderly Lung Cancer Vinorelbine Italian Study Group: Effects of vinorelbine on quality of life and survival of elderly patients with advanced non-small-cell lung cancer. J Nat/ Cancer Inst 1999, 91(1):66-72.

31. Gridelli C: The ELVIS trial: a phase III study of single-agent vinorelbine as first-line treatment in elderly patients with advanced non-small cell lung cancer. Elderly Lung Cancer Vinorelbine Italian Study. Oncologist 2001, 6 (Suppl 1):4-7.

32. Gridelli C, Perrone F, Gallo C, Cigolari S, Rossi A, Piantedosi F, Barbera S, Ferrau F, Piazza E, Rosetti F, et al: Chemotherapy for elderly patients with advanced non-small-cell lung cancer: the Multicenter Italian Lung Cancer in the Elderly Study (MILES) phase III randomized trial. J Natl Cancer Inst 2003, 95(5):362-372.

33. Quoix EA, Oster J, Westeel V, Pichon E: Weekly paclitaxel combined with monthly carboplatin versus single-agent therapy in patients age 70 to 89: IFCT-0501 randomized phase III study in advanced non-small cell lung cancer (NSCLC). In: J Clin Oncol 2010, 2010(suppl; abstr 2):18s.

\section{doi:10.1186/1471-2407-12-185}

Cite this article as: Fujita et al:: Customized chemotherapy based on epidermal growth factor receptor mutation status for elderly patients with advanced non-small-cell lung cancer: a phase II trial. BMC Cancer 2012 12:185. 